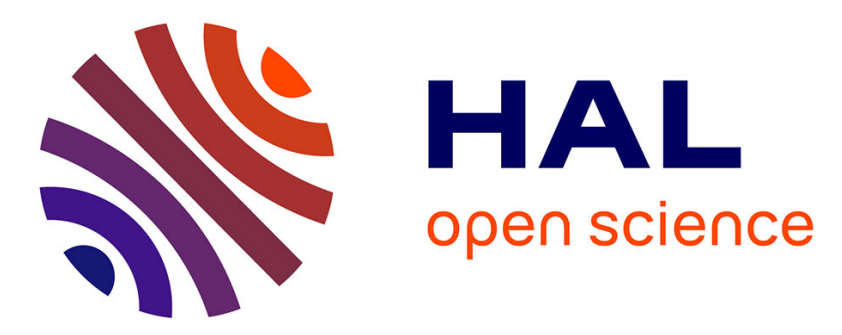

\title{
Intra and Inter-Observer Reliability of Determining Degree of Pelvic Obliquity in Neuromuscular Scoliosis Using the EOS-CHAIR® Protocol
}

Jihane Rouissi, Robin Arvieu, Arnaud Dubory, Claudio Vergari, Manon Bachy, Raphaël Vialle

\section{To cite this version:}

Jihane Rouissi, Robin Arvieu, Arnaud Dubory, Claudio Vergari, Manon Bachy, et al.. Intra and InterObserver Reliability of Determining Degree of Pelvic Obliquity in Neuromuscular Scoliosis Using the EOS-CHAIR® Protocol. Child's Nervous System, 2017, 33 (2), pp.337-341. 10.1007/s00381-0163326-5 . hal-02890095

\section{HAL Id: hal-02890095 https://hal.science/hal-02890095}

Submitted on 6 Jul 2020

HAL is a multi-disciplinary open access archive for the deposit and dissemination of scientific research documents, whether they are published or not. The documents may come from teaching and research institutions in France or abroad, or from public or private research centers.
L'archive ouverte pluridisciplinaire HAL, est destinée au dépôt et à la diffusion de documents scientifiques de niveau recherche, publiés ou non, émanant des établissements d'enseignement et de recherche français ou étrangers, des laboratoires publics ou privés. 


\title{
Intra and inter-observer reliability of determining degree of pelvic obliquity in neuromuscular scoliosis using the EOS-CHAIR ${ }^{\circledR}$ protocol
}

\author{
Jihane Rouissi $^{1}$ • Robin Arvieu ${ }^{1}$ - Arnaud Dubory ${ }^{1,2}$ - Claudio Vergari ${ }^{3,4}$. $^{1}$ \\ Manon Bachy ${ }^{1,2} \cdot$ Raphaël Vialle ${ }^{1,2}$
}

\begin{abstract}
Purpose Scoliosis with pelvic obliquity (PO) could be investigated with the EOS-CHAIR protocol as the most common deformity especially in patients with trunk hypotonia and quadriplegia. However, the intra-observer and inter-observer reliability of various angles assessing PO was not investigated with this new imaging protocol.

Methods A retrospective cohort of 36 EOS frontal fullspine acquisitions made in sitting position was used. The sacroiliac pelvic obliquity angle, iliac crest pelvic obliquity angle, and ischiatic pelvic obliquity angle were assessed in an intra-observer and inter-observer study.

Results The use of the EOS-CHAIR protocol was implemented satisfactory with a high acceptance rate by all caregivers and patients and their families. Intra-observer and inter-observer reliability was excellent for the three tested angular measurements.

Discussion As for idiopathic scoliosis, we postulate the EOS system as being superior to standard radiographs to
\end{abstract}

Raphaël Vialle

raphael.vialle@aphp.fr

1 Department of Pediatric Orthopaedics, Armand Trousseau Hospital, Université Pierre et Marie Curie Paris, 26 avenue du Dr Arnold Netter, 75571Cedex 12 Paris, France

2 Department for Innovative Therapies in Musculoskeletal Diseases Armand Trousseau Hospital, The MAMUTH Hospital-University, 26 avenue du Docteur Arnold Netter, F-75571 Cedex12 Paris, France

3 Arts et Métiers, Paristech, Institut de Biomécanique Humaine Georges Charpak, 151 Boulevard de l'Hôpital, 75013 Paris, France

4 School of Physics, University of Exeter, Stocker Road, Exeter EX4 4QL, UK assess 3D spinal deformities in neuromuscular conditions. The EOS-CHAIR protocol improves preoperative comprehension of the lumbosacral junction anatomy in patients with poor standing or sitting postures. Our results show a very high reliability of three different angular measurements of the frontal pelvic obliquity in sitting position. Then it is possible to use one of these three angles as well as the others to assess frontal pelvic obliquity in neuromuscular patients. This frontal pelvic obliquity protocol in sitting position with the EOS-CHAIR is a validated measurement technique that needs to be used now to measure PO as a critical parameter of the global trunk balance in neuromuscular patients.

Keywords Neuromuscular scoliosis · Eos-chair · Pelvic obliquity $\cdot$ Trunk imbalance $\cdot$ Eos system $\cdot$ CD reformatting

\section{Introduction}

Spinal deformity is common in children and adolescents with neuromuscular disorders. Incidence is higher than 20 to $25 \%$ in non-ambulatory patients and total-body cerebral palsy (CP) patients [1]. Scoliosis with pelvic obliquity (PO) is the most common deformity especially in patients with trunk hypotonia and quadriplegia. The development of a severe trunk deformity associated with the inherent neurological disorder can significantly restrict the patients' functional capacities and maximize the need for nursing care [1-3]. Sitting tolerance may be increasingly diminished and trunk decompensation can result in back or rib impingement pain, as well as pulmonary complications [4]. Such patients often require a long fusion from the upper thoracic spine to the sacrum or pelvis to achieve and maintain correction of the curve and the PO. One of 
the technical challenges of such surgical strategy is the objective evaluation and measurement of pelvic obliquity in sitting position. Previous published data established that the EOS system 2D/3D images made with the EOSCHAIR protocol improve preoperative comprehension of the lumbosacral junction anatomy and assessment of the global frontal and sagittal sitting balance [5]. However, the intra-observer and inter-observer reliability of various angles assessing PO was not investigated with this new imaging protocol. The aim of this study was to assess the reliability of various PO angular measurements and to propose the best technique to validate the best way to evaluate this very critical radiological data with the EOS-CHAIR solution.

\section{Material and methods}

This study was retrospective, using a cohort of 36 EOS frontal full-spine acquisitions made in neuromuscular patients with pelvic obliquity. The EOS-CHAIR protocol [5] was used as follows: The patient was placed in the chair by two persons (the parents with the help of radiology department caregivers). Then, the three-point seatbelts were adjusted and closed to prevent accidental fall from the chair. The headrest was adjusted to the appropriate height. The headbands were used for children with poor cervical tonus. Once patients were properly seated, the chair was introduced into the EOS system by its $46-\mathrm{cm}$-wide access and placed for image acquisition. During image acquisition (approximately $20 \mathrm{~s}$ ), patients were required to have their arms in upper position. At the end of the procedure, the chair is pulled backward from the EOS system using its handling straps. With this protocol, it was possible to visualize the whole axial skeleton between the head and the pelvis. PO was assessed using three different angular measurements (Fig. 1):

- The sacroiliac pelvic obliquity angle $[6,7]$ (SIPOA) was measured by defining a sacroiliac line between the inferior edges of both sacroiliac joints on the frontal radiograph, the perpendicular to this line, and the spinous process of $\mathrm{T} 1$ to the middle of the sacroiliac line (Fig. 1a).

- The iliac crest pelvic obliquity angle (ICPOA) was measured by defining an iliac crest line between the upper part of both iliac crests on the frontal radiograph, the perpendicular to this line, and the spinous process of $\mathrm{T} 1$ to the middle of the iliac crest line (Fig. 1b).

- The ischiatic pelvic obliquity angle (IPOA) was measured by defining an ischiatic line between the lower part of both ischiatic bones on the frontal radiograph,
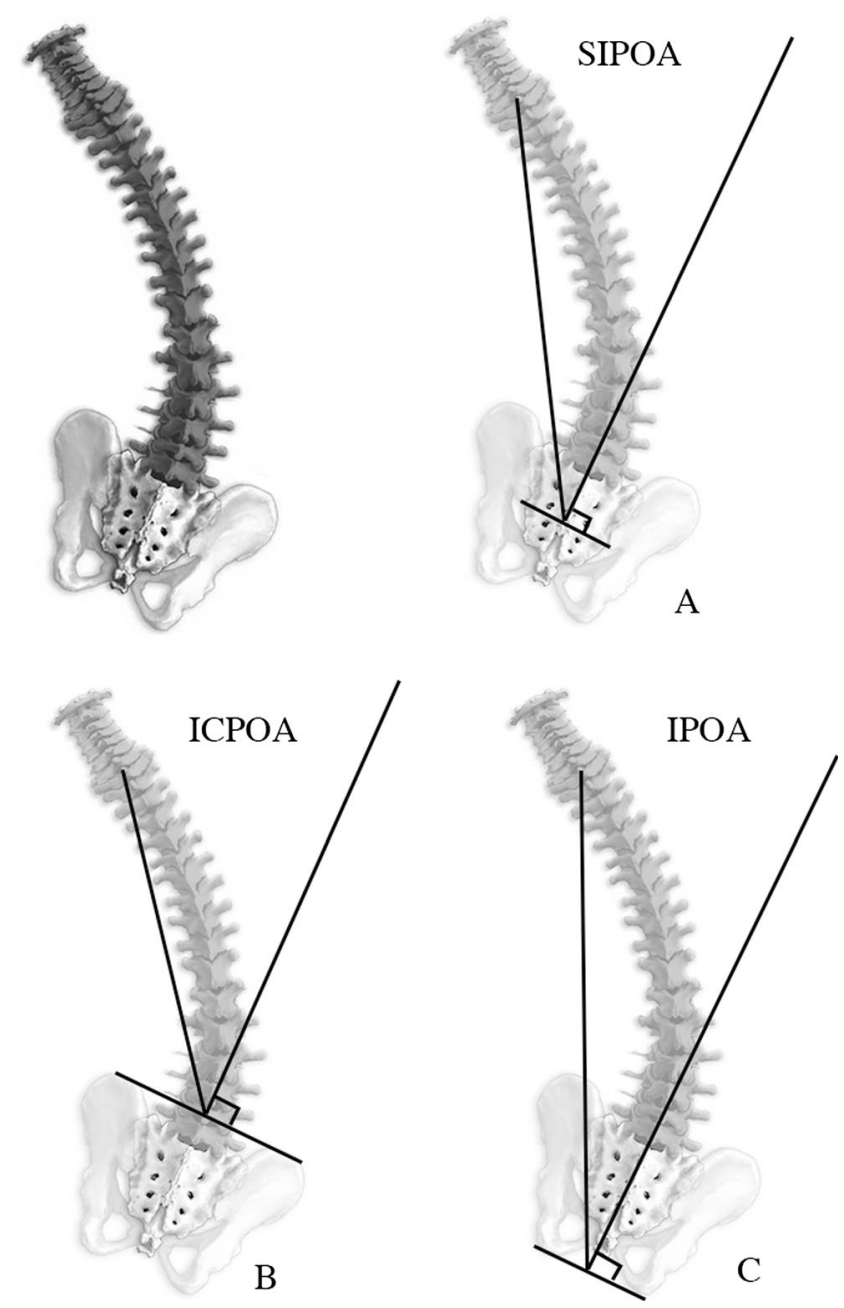

Fig. 1 The three different angles used to assess the frontal pelvic obliquity. a The sacroiliac pelvic obliquity angle (SIPOA) was measured by defining a sacroiliac line between the inferior edges of both sacroiliac joints on the frontal radiograph, the perpendicular to this line, and the spinous process of $\mathrm{T} 1$ to the middle of the sacroiliac line. $\mathbf{b}$ The iliac crest pelvic obliquity angle (ICPOA) was measured by defining an iliac crest line between the upper part of both iliac crests on the frontal radiograph, the perpendicular to this line, and the spinous process of T1 to the middle of the iliac crest line. $\mathbf{c}$ The ischiatic pelvic obliquity angle (IPOA) was measured by defining an ischiatic line between the lower part of both ischiatic bones on the frontal radiograph, the perpendicular to this line, and the spinous process of $\mathrm{T} 1$ to the middle of the ischiatic line

the perpendicular to this line, and the spinous process of T1 to the middle of the ischiatic line (Fig. 1c).

First, the intra- and inter-observer reliability of the threeangle measurements were assessed. Computer-assisted measurements of the three different angles were made in the 36 cases by two different observers (JR and RA), on two separate occasions, in random order using the same software (Carestream PACS Carestream Health, Inc. 150 Verona Street, Rochester, NY, 14608, USA). For all comparisons, the reliability between the two series of measures was assessed using Spearman's rank correlation test, a $t$ test and intraclass 
correlation coefficient (ICC) [8], and the limits of agreement by Bland and Altman [9]. The data were analyzed with SPSS V22.0 software (SPSS Inc. Chicago, IL, USA). A $P$ value for Spearman's rank correlation and all $t$ tests were considered significant if less than 0.05 . Intraclass correlation coefficients (ICC) [8] of one imply perfect agreement, and values less than one imply less than perfect agreement [8].

\section{Results}

The use of the EOS-CHAIR protocol was implemented satisfactory with a high acceptance rate by all caregivers and patients and their families. We did not experience adverse effects using this technique. The quality of the EOS images allowed assessing preoperative trunk collapse and pelvic obliquity as well as postoperative corrections in all planes (Fig. 2).

\section{Intra-observer reproducibility}

Intra-observer reproducibility of the manual method Spearman's rank correlation showed significant correlation between data sets for ischiatic pelvic obliquity angle (IPOA) $(R=0.913, P<0.0001)$ and the paired $t$ test was again significant $(t=0.913, P<0.0001)$. The ICC was $0.954(0.927-$ 0.972), and the graphic study by Altman and Bland showed a statistically significant difference between two measurements out of 72 .

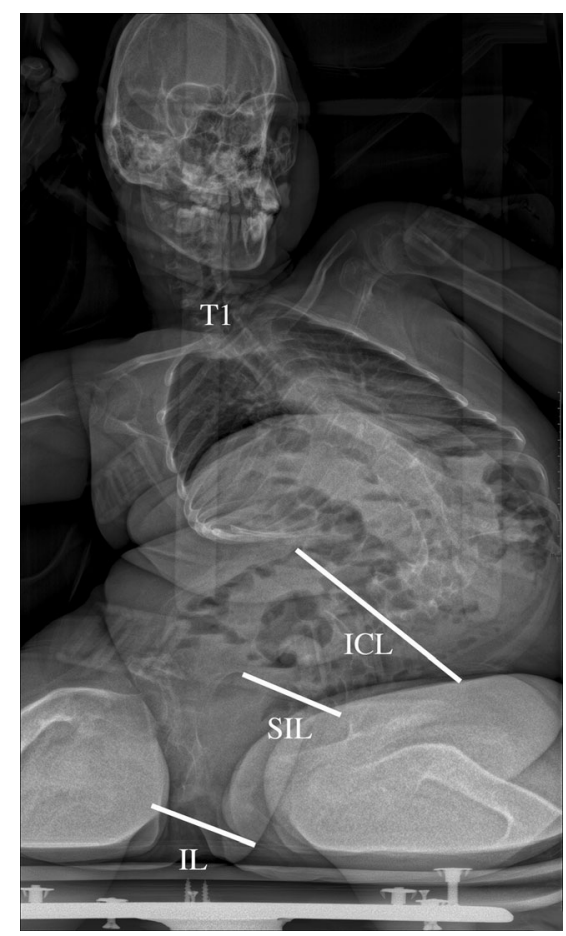

Fig. 2 Example of a young patient with a severe trunk deformity due to cerebral palsy. The EOS-CHAIR protocol gives the opportunity to visualize trunk deformity and pelvic obliquity in sitting position
Intra-observer reproducibility of the manual method Spearman's rank correlation showed significant correlation between data sets for sacroiliac pelvic obliquity angle (SIPOA) $(R=0.932, P<0.0001)$ and the paired $t$ test was again significant $(t=0.955, P<0.0001)$. The ICC was 0.977 (0.964-0.986), and the graphic study by Altman and Bland showed a statistically significant difference between one measurement out of 72 .

Intra-observer reproducibility of the manual method Spearman's rank correlation showed significant correlation between data sets for iliac crest pelvic obliquity angle (ICPOA) $(R=0.931, P<0.0001)$ and the paired $t$ test was again significant $(t=0.955, P<0.0001)$. The ICC was 0.976 (0.962-0.985), and the graphic study by Altman and Bland showed a statistically significant difference between one measurement out of 72 .

\section{Inter-observer reproducibility}

Inter-observer reproducibility of the manual method Spearman's rank correlation showed significant correlation between data sets for ischiatic pelvic obliquity angle (IPOA) $(R=0.771$, $P<0.0001)$ and the paired $t$ test was again significant $(t=0.820, P<0.0001)$. The ICC was $0.898(0.927-0.972)$ and the graphic study by Altman and Bland showed a statistically significant difference between two measurements out of 72 .

Inter-observer reproducibility of the manual method Spearman's rank correlation showed significant correlation between data sets for sacroiliac pelvic obliquity angle (SIPOA) $(R=0.845, P<0.0001)$ and the paired $t$ test was again significant $(t=0.898, P<0.0001)$. The ICC was 0.944 (0.910-0.965), and the graphic study by Altman and Bland showed a statistically significant difference between two measurements out of 72 .

Inter-observer reproducibility of the manual method Spearman's rank correlation showed significant correlation between data sets for iliac crest pelvic obliquity angle (ICPOA) $(R=0.868, P<0.0001)$ and the paired $t$ test was again significant $(t=0.932, P<0.0001)$. The ICC was 0.965 (0.944-0.978), and the graphic study by Altman and Bland showed a statistically significant difference between one measurement out of 72 .

The summary of statistical analysis is reported in Table 1.

\section{Discussion}

Scoliosis with pelvic obliquity (PO) is the most common deformity especially in patients with trunk hypotonia and quadriplegia [3]. Progressive spinal deformity causes difficulty with daily care, walking, and sitting and can lead to back and rib pain, cardiac and pulmonary complications, altered seizure thresholds, and skin compromise [10]. Interest in the 
Table 1 Summary of statistical data

\begin{tabular}{lllll}
\hline & Pair tested & Test used & Value & $P$ value \\
\hline Intra-observer reliability & \multirow{2}{*}{ IPOA } & Spearman's rho & 0.771 & $<0.0001$ \\
& & Paired $t$ test & 0.820 & $<0.0001$ \\
& SIPOA & Intraclass correlation coefficient & 0.898 & \\
& & Spearman's rho & 0.845 & $<0.0001$ \\
& Paired $t$ test & 0.898 & $<0.0001$ \\
& ICPOA & Intraclass correlation coefficient & 0.944 & \\
& & Spearman's rho & 0.931 & $<0.0001$ \\
& & Paired $t$ test & 0.955 & $<0.0001$ \\
& Inter-observer reliability & Intraclass correlation coefficient & 0.976 & \\
& & Spearman's rho & 0.771 & $<0.0001$ \\
& & Paired $t$ test & 0.820 & $<0.0001$ \\
& \multirow{2}{*}{ SIPOA } & Intraclass correlation coefficient & 0.898 & \\
& & Spearman's rho & 0.845 & $<0.0001$ \\
& & Paired $t$ test & 0.898 & $<0.0001$ \\
& & Intraclass correlation coefficient & 0.944 & \\
& ICPOA & Spearman's rho & 0.868 & $<0.0001$ \\
& & Paired $t$ test & 0.932 & $<0.0001$ \\
& & Intraclass correlation coefficient & 0.965 & \\
& & &
\end{tabular}

potential clinical relevance and impact of 3D analysis of trunk and chest deformities is growing. Formerly, CT scanning was the most frequently used imaging tool for such 3D analysis but at the expense of high radiation exposure, and with the limitation of being performed in the supine position [11]. These drawbacks can be avoided in ambulatory patients by the use of the EOS system low-dose biplanar radiographs. In standing adolescent idiopathic scoliosis patients, the validity of routine $3 \mathrm{D}$ reconstructions has been established at present [12]. As for idiopathic scoliosis, we postulate the EOS system as being superior to standard radiographs to assess 3D spinal deformities in neuromuscular conditions. Thanks to our EOS-CHAIRvalidated protocol [5], the EOS system 2D/3D images improve preoperative comprehension of the lumbosacral junction anatomy in patients with poor standing or sitting postures. However, as PO is commonly associated with scoliosis in cerebral palsy making sitting difficult [6], we consider as crucial to investigate pelvis positioning in sitting position. Our results show a very high reliability of three different angular measurements of the frontal pelvic obliquity in sitting position. Compared to the well-known sacroiliac pelvic obliquity angle $[6,7]$, the angular measurements based on ischiatic and iliac crest landmarks are also reliable and reproducible. Then it is possible to use one of these three angles as well as the others to assess frontal pelvic obliquity in neuromuscular patients. This frontal pelvic obliquity protocol in sitting position with the EOS-CHAIR is a validated measurement technique that needs to be used now to measure PO as a critical parameter of the global trunk balance in neuromuscular patients. In further clinical studies, we stress the importance of such simple and fast technique in saving time and maximizing inter- and intra-observer repeatability of angular measurements. We fully recognize that the time gain was not clearly studied in the current study, but only a few minutes were necessary to obtain the three PO values. Despite ischiatic landmarks may be more difficult to identify in some patients, iliac crest or sacroiliac lines could be used without any risk in term of reliability. Additionally, these three easy ways to evaluate the frontal pelvic obliquity could be useful to design promising $3 \mathrm{D}$ pelvis reformatting tools to be associated with $3 \mathrm{D}$ spine reformatting models in the future.

\section{Compliance with ethical standards}

Conflict of interest Jihane Rouissi: No conflict of interest to disclose. Robin Arvieu: No conflict of interest to disclose. Arnaud Dubory: No conflict of interest to disclose. Claudio Vergari: No conflict of interest to disclose. Manon Bachy: No conflict of interest to disclose. Raphaël Vialle works as a consultant for Stryker Spine

\section{References}

1. Anari JB, Spiegel DA, Baldwin KD (2015) Neuromuscular scoliosis and pelvic fixation in 2015: where do we stand? World J Orthop 6:564-566. doi:10.5312/wjo.v6.i8.564

2. Auerbach JD, Spiegel DA, Zgonis MH, et al. (2009) The correction of pelvic obliquity in patients with cerebral palsy and neuromuscular scoliosis: is there a benefit of anterior release prior to posterior 
spinal arthrodesis? Spine Phila Pa 1976 34:E766-74. doi:10.1097 /BRS.0b013e3181b4d558

3. Vialle R, Thevenin-Lemoine C, Mary P (2013) Neuromuscular scoliosis. Orthop Traumatol Surg Res 99:S124-S139. doi:10.1016 /j.otsr.2012.11.002

4. Brooks JT, Sponseller PD (2015) What's new in the management of neuromuscular scoliosis. J Pediatr Orthop. doi:10.1097 /BPO.0000000000000497

5. Bouloussa H, Dubory A, Seiler C et al (2015) A radiolucent chair for sitting-posture radiographs in non-ambulatory children: use in biplanar digital slot-scanning. Pediatr Radiol 45:1864-1869. doi:10.1007/s00247-015-3374-0

6. Bouyer B, Bachy M, Zahi R et al (2014) Correction of pelvic obliquity in neuromuscular spinal deformities using the "T construct": results and complications in a prospective series of 60 patients. Eur Spine J 23:163-171. doi:10.1007/s00586-013-2847-9

7. Vialle R, Delecourt C, Morin C (2006) Surgical treatment of scoliosis with pelvic obliquity in cerebral palsy: the influence of intraoperative traction. Spine Phila Pa 1976 31:1461-6. doi:10.1097/01.brs.0000219874.46680.87

8. Lin LI (1989) A concordance correlation coefficient to evaluate reproducibility. Biometrics 45:255-268

9. Bland JM, Altman DG (1990) A note on the use of the intraclass correlation coefficient in the evaluation of agreement between two methods of measurement. Comput Biol Med 20:337-340

10. Roberts SB, Tsirikos AI (2016) Factors influencing the evaluation and management of neuromuscular scoliosis: a review of the literature. J Back Musculoskelet Rehabil. doi:10.3233/BMR-160675

11. Melhem E, Assi A, El Rachkidi R, Ghanem I (2016) EOS(®) biplanar X-ray imaging: concept, developments, benefits, and limitations. J Child Orthop 10:1-14. doi:10.1007/s11832-0160713-0

12. Courvoisier A, Garin C, Vialle R, Kohler R (2015) The change on vertebral axial rotation after posterior instrumentation of idiopathic scoliosis. Childs Nerv Syst ChNS off J Int Soc. Pediatr Neurosurg 31:2325-2331. doi:10.1007/s00381-015-2891-3 